\title{
MicroCorrespondence
}

\section{Structure of the DNA-binding domain of the OmpR family of response regulators}

Sir,

The Escherichia coli DNA-binding protein, OmpR, is one of the best characterized of the bacterial positive regulators that enhance the transcriptional ability of RNA polymerase. Furthermore, OmpR is a typical example of response regulators involved in the widespread two-component phosphotransfer signalling systems (Parkinson and Kofoid, 1992, Annu Rev Genet 26: 71-112). OmpR contains a phospho-accepting receiver domain of approx. 120 amino acids at the $\mathrm{N}$-terminal portion, followed by a Cterminal DNA-binding domain of approx. 120 amino acids. An inspection of current databases (SWISSPROT/PIR/ GenBank) revealed that $E$. coli has at least 15 members of the OmpR family of proteins, including one encoded by an E. coli plasmid, whose amino acid sequences show extensive similarities to each other in their C-terminal DNA-binding domains as well as the $\mathrm{N}$-terminal receiver domains. The occurrence of members of the OmpR family has also been reported frequently for many other bacterial species. These facts suggest that the OmpR-like proteins are one of the most widespread DNA-binding transcriptional regulators in bacteria.

The members of the OmpR family in $E$. coli and related species have long been the subject of debate in this journal (OmpR, Mizuno and Mizushima, 1990, Mol Microbiol 4: 1077-1082; KdpE, Nakashima et al., 1993, Mol Microbiol 7: 109-116; PhoP, Wick et al., 1995, Mol Microbiol 16: 465-47; ArcA, Drapal and Sawers, 1995, Mol Microbiol 16: 597-607; TorR, Simon et al., 1995, Mol Microbiol 17: 971-980). One of the crucial issues concerning the OmpR family of proteins is the molecular mechanism underlying DNA recognition. A previous inspection of the primary structure of OmpR failed to find a canonical helix-turn-helix (HTH) DNA-binding motif, which is commonly found in a number of $E$. coli DNA-binding proteins. Furthermore, these OmpR-like proteins are highly similar to each other in their presumed DNA-binding domains, yet they are postulated to regulate (activate or repress) specific subsets of genes in E. coli. The other interesting issue is the underlying molecular mechanism of gene activation per se. It has been suggested that the DNA-binding domain of OmpR contains a direct contact site(s) with RNA polymerase (particularly with the $\alpha$-subunit). The threedimensional structure of OmpR, which would help us study these relevant issues in more depth, is a long time in coming. The structure of the C-terminal DNA-binding domain of OmpR, determined by X-ray crystallography, is now available (Kondo et al., 1997, Nature Struct Biol 4: 28-31; Martinez-Hackert and Stock, 1997, Structure 5: 109-124). The determined secondary structure of the Cterminal domain of OmpR (residues 137-234) is shown schematically in Fig. 1. This structural view also allows us to unambiguously align the sequences of all the $E$. coli OmpR family, and should be useful, particularly for studying the OmpR family of $E$. coli and other species (note that the additional member, $\mathrm{CadC}$, is atypical in the sense that it does not belong to the family of response regulators).

With special reference to the DNA recognition by the OmpR family of proteins, the $\mathrm{X}$-ray structure reveals that two helices, $\alpha 2$ and $\alpha 3$, create a structure that superimposes well on those of other canonical HTH motifs (e.g. CRP, cAMP receptor protein). However, this presumed HTH structure of OmpR is unique in the sense that it has a long loop between $\alpha 2$ and $\alpha 3$. This may be the reason why previous surveys failed to detect a putative HTH motif in OmpR. One can now envisage that $\alpha 3$ of OmpR is the DNA-recognition helix, as is the case for the standard HTH-type DNA-binding proteins.

This presumed recognition helix, $\alpha 3$, consisting of 12 amino acid residues, is enlarged in Fig. 2, along with those of other members of the OmpR family of proteins. It is clear that hydrophobic residues (hydrophobic a.a.) at positions 1, 5, 8 and 12 are well conserved among the OmpR protein family (Fig. 2, left-hand panel). When these residues are depicted on an $\alpha$-helical wheel, they create a hydrophobic face along one side of the helical cylinder (Fig. 2, right-hand panel). In the determined structure of OmpR, this hydrophobic side is facing the protein core. If $\alpha 3$ is the DNA-recognition helix, as presumed, other residues in $\alpha 3$ may be facing DNA (or bases in the major groove). Residues at positions 7, 9 and 11 are frequently positively charged amino acids (+a.a.). As indicated in Fig. 2, these residues on the equator may be involved in an interaction with the negatively charged DNA backbones. The remaining residues, at positions 3,6 and 10, may be in close proximity to specific bases, thereby enabling OmpR to recognize a specific target DNA. The DNAbinding specificity for each member may be determined, at least in part, by these amino acids (note that the amino acid combination in OmpR, i.e. Val-3, Ser-6 and Arg-10, is not identical to the amino acid combination 


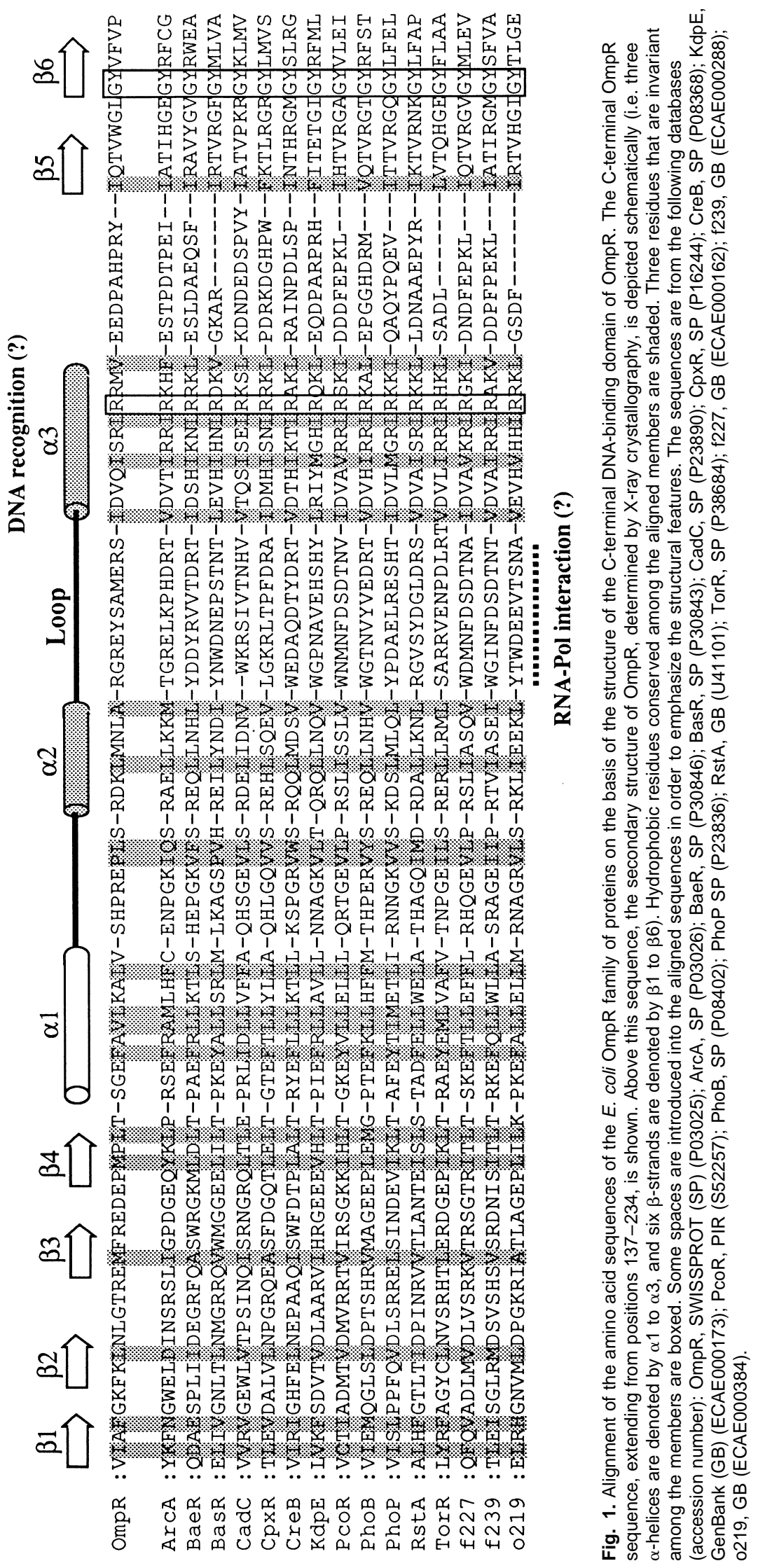



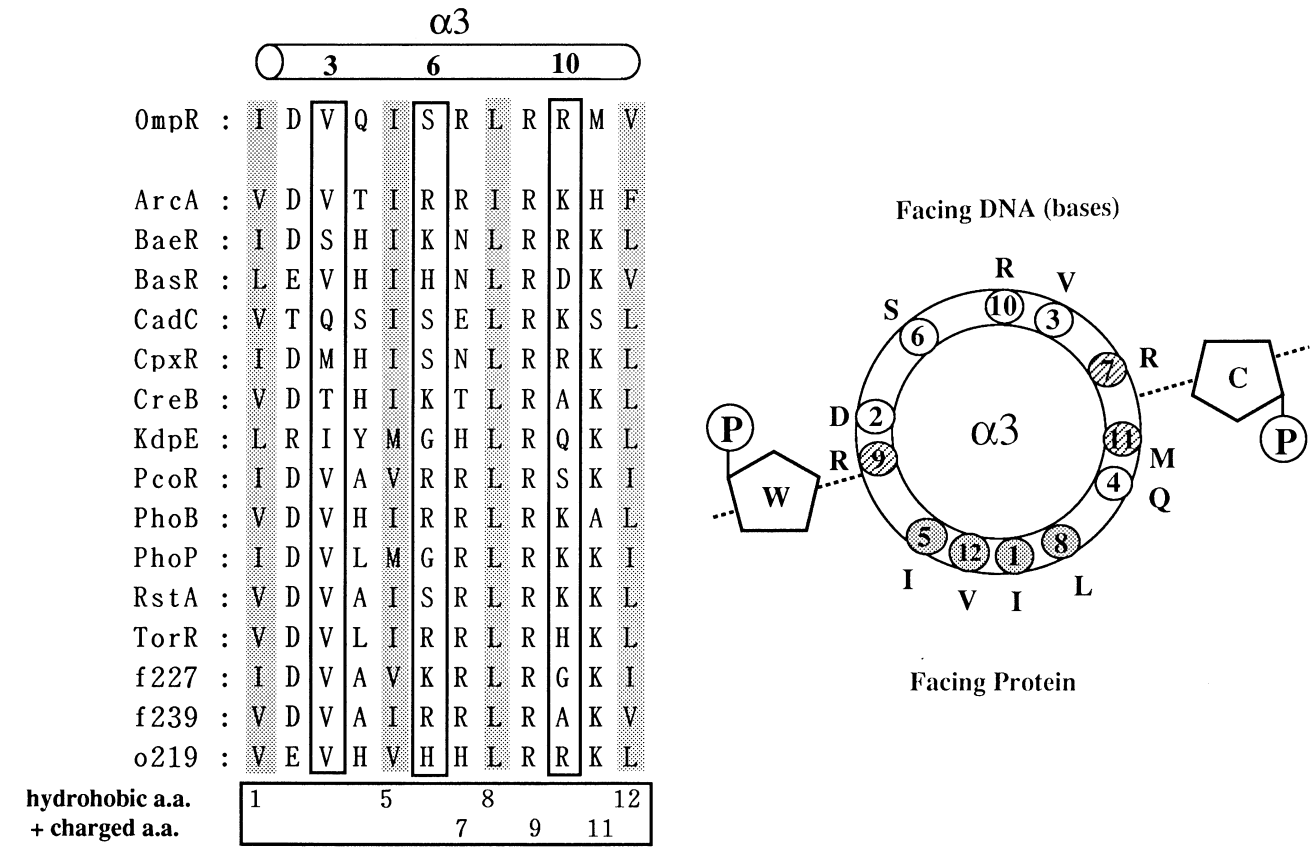

Fig. 2. The putative DNA-recognition helix in the C-terminal DNA-binding domain of OmpR. The $\alpha 3$ sequence of the putative DNA-recognition helix of OmpR is shown, along with those of other members of the OmpR family. The $\alpha 3$ sequence, consisting of 12 amino acid residues, is also plotted in an $\alpha$-helical wheel. This helix is supposed to interact with the major groove of DNA. (DNA backbones are indicated by pentagons; W, Watson strand; C, Crick strand.) Other details are given in the text.

found at these positions in any other members). It may also be worth mentioning that a classical OmpR mutant, OmpR2, which exhibits an OmpF-constitutive and OmpCnegative phenotype, turned out to have an amino acid substitution at position 3 (Val-203 $\rightarrow$ Met).

Regarding the contact domain with RNA polymerase, a set of positive control (PC) mutants have previously been reported for OmpR (Pratt and Silhavy, 1994, J Mol Biol 243: 579-594; Koto et al., 1995, Mol Gen Genet 248: 399-406). On the basis of the determined structure, the amino acid substitutions in these PC-mutants are located in the loops between $\alpha 2$ and $\alpha 3$ (Glu-193, Ala-196, Glu198), or $\alpha 1$ and $\alpha 2$ (Pro-179, Ser-181) (see Fig. 1). Such loop regions in the OmpR family of proteins are thus considered to be involved in an interaction with RNA polymerase (either with the $\alpha$ - or $\sigma$-subunit).

In conclusion, a close inspection of these amino acid sequences of OmpR-like proteins, in the context of the determined OmpR structure, should provide us with new and at least preliminary insights into the structure and function of the members of the large OmpR family of proteins.

Takeshi Mizuno $^{1 *}$ and Isao Tanaka ${ }^{2}$

${ }^{1}$ Laboratory of Molecular Microbiology, School of

Agriculture, Nagoya University, Chikusa-ku, Nagoya 464, Japan.

${ }^{2}$ Division of Biological Sciences, Graduate School of Science, Hokkaido University, Sapporo 060, Japan.

* For correspondence.

E-mail i45455a@nucc.cc.nagoya-u.ac.jp; Tel. (52) 789 4089. Fax (52) 7894091.

Received 5 December, 1996; revised 18 February, 1997; accepted 26 February, 1997

\section{Neisseria gonorrhoeae reverse transcriptase activity does not mediate pilin gene conversion}

Sir,

Pilin variation in Neisseria gonorrhoeae is implicated in both immune evasion and modulation of the bacterial adherence to human host cells. Frequent changes in the main subunit of the pili are caused by homologous recombination events between the expression locus pilE and several variant silent pilin genes (pilS) lacking a promoter and 5 '-terminal codons. Pilin variation can occur by either transformation or intra-strain recombination (for a review see Robertson and Meyer, 1992, Trends Genet 8: 422427; Seifert, 1996, Mol Microbiol 21: 433-440). Intriguingly, the latter pathway usually results in non-reciprocal, 


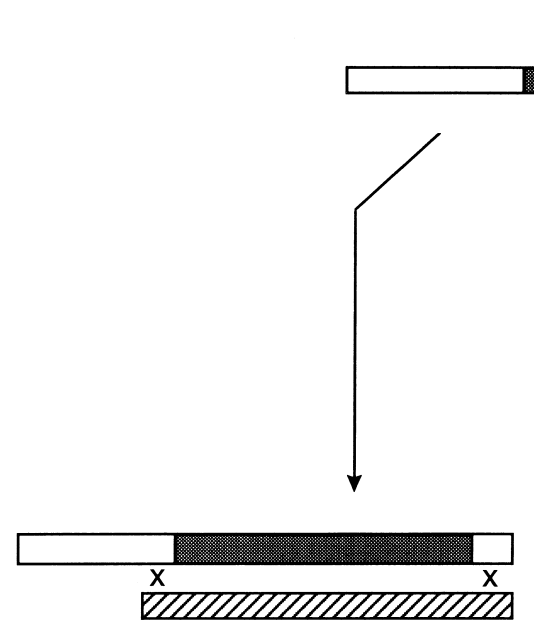

Self-splicing intron

ص pils

Antisense

transcription

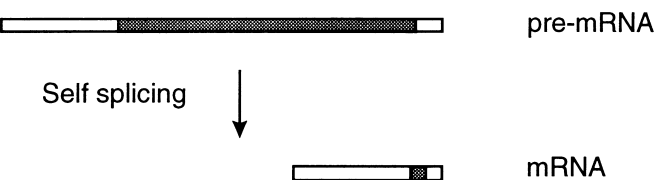

Reverse
transcription $\downarrow$

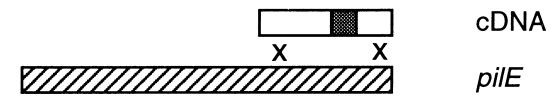

pilE

Homologous
recombination
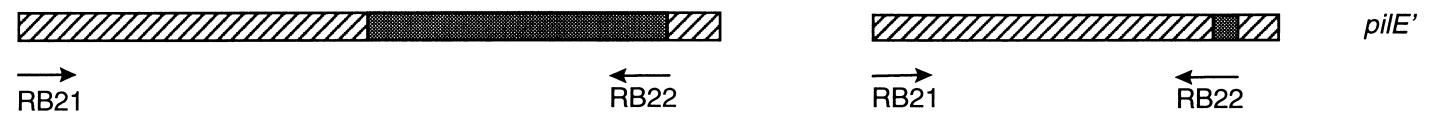

Fig. 1. Hypothetical pathways that could account for non-reciprocal recombination (gene conversion) with pilE1. The donor locus (pilS) was modified so that it contains a self-splicing intron. If gene conversion involves the formation of a precursor transcript (pre-mRNA) of pilS, self-splicing should occur and recombination of the resulting cDNA should result in a small insertion in pilE (right-hand panel). If no RNA intermediate exists, gene conversion should lead to a large insertion in pilE (left-hand panel). Using the primer pair RB21

(5'-GGCTTTCCCCTTTCAATTAGGAG-3') and RB22 (5'-TCCGAATTCCCACTCTCT-3') for PCR, specific gene-conversion events between the pilS1 and pilE1 could be readily detected and the outlined recombination pathways distinguished.

gene-conversion-like changes. In yeast cells, gene-conversion events can be mediated by the synthesis of a diffusible reverse transcript and its subsequent recombination into the chromosome (Derr and Strathern, 1993, Nature 361: 170-173). In addition, prokaryotic reverse transcriptases (RTs) are able to synthesize double-stranded DNA (Lampson et al., 1990, J Biol Chem 265: 8490-8496), and homologous recombination can be promoted by RT activity in vitro (Negroni et al., 1995, Proc Natl Acad Sci USA 92: 6971-6975). We thus addressed the questions of whether gonococci possess detectable RT activity and if the observed gene conversions are mediated by reverse transcription.

The soluble fraction obtained from bacterial lysates (Brutlag et al., 1971, Proc Natl Acad Sci USA 68: 28262829) was assessed for RT activity using the highly specific assay described by Silver et al. (1993, Nucleic Acids Res 21: 3593-3594). Single-stranded Brome Mosaic Virus (BMV) RNA (Promega) was reverse transcribed to yield cDNA using a specific primer, and then amplified via a polymerase chain reaction (PCR) using two other primers derived from the BMV sequence. Boiled cell extracts served as a negative control, whereas avian myeloblastosis virus (AMV) RT (USB) was used as positive control for the reverse transcription of the RNA into DNA. Both untreated gonococcal extracts and purified Escherichia coli DNA polymerase I (Pharmacia) yielded the predicted PCR product which is indicative of RT activity (data not shown). Whether this measured RT activity is mediated by a gonococcal DNA polymerase I or by a distinct RT is, at present, unknown. Multicopy single-stranded DNA (msDNA) associated with prokaryotic reverse transcriptase activity has previously not been detected in gonococci (Innouye and Innouye, 1991, Annu Rev Microbiol 45: 163-186).

In order to test the hypothesis that gene conversion in gonococci is based on the reverse transcription of pilS genes, we developed a genetic system to monitor such events. N969, a derivative of $N$. gonorrhoeae strain MS11, was mutated in $\operatorname{com} A$, leading to transformation deficiency so that transformation-mediated pilin variation was excluded (Facius and Meyer, 1993, Mol Microbiol 10: 699-712). A cloned invariant pilC gene (Rudel et al., 1995, Nature 373: 357-359) ensured that switching between piliated $\left(\mathrm{P}^{+}\right)$ and non-piliated $\left(\mathrm{P}^{-}\right)$phenotypes was not due to phase variation of PilC expression. Furthermore, a stop signal, an RB22-primer-binding site, and a self-splicing intron from Tetrahymena (generously provided by R. B. Waring 
(Waring, 1989, Nucleic Acids Res 17: 10281-10293)) were cloned into the hypervariable region (mc2) (Haas et al., 1992, Mol Microbiol 6: 197-208) of pilS1 copy1. Constructs were verified by sequencing. The mc2 region was chosen because gene-conversion events involving this region are well documented, and copy 1 of pilS1 was chosen because evidence exists for a role of the adjacent Sma/Cla repeat in pilin gene recombination (Wainwright et al., 1994, Mol Microbiol 13: 75-78). Introduction of the construct into the gonococcal chromosome was selected for by using a chloramphenicol-resistance gene (cat) flanked by directly repeated internal resolution RES sites of the transposon Tn3. After chromosomal insertion, the cat cassette was excised by chromosomal integration of the resolvase $(\operatorname{tnp} R)$ gene of transposon Tn3 into the chromosomal iga gene. Finally, by allelic exchange, $\operatorname{tnp} R$ was replaced with a kanamycin-resistance gene. Gene-conversion events were monitored on the basis of colony-morphology changes which are coupled to $\mathrm{P}^{+}$-to$\mathrm{P}^{-}$transition events. The events were further analysed by PCR, employing primer RB21, which binds specifically to the ribosome-binding site of pilE but not to the pilS regions, and primer RB22. The Tetrahymena intron is spliced from a mRNA precursor (pre-mRNA) both in vitro and in E. coli (Waring et al., 1985, Cell 40: 371-380). If gene conversion is mediated by a diffusible reverse transcript, the self-splicing event ought to be readily detected because the resulting cDNA would thus lack the intron sequence (Fig. 1). Because the splicing reaction occurs only from plus-strand mRNA, the intron has been inserted in both transcriptional orientations. Although more than 100 $\mathrm{P}^{+}$-to- $\mathrm{P}^{-}$switch variants possessing the intron in either orientation were analysed that have undergone gene conversions from pilS1 to pilE1, no clones harbouring spliced introns in pilE1 were detected. This strongly suggests that gene conversion in Neisseria does not involve a mRNA intermediate of the donor pilS gene.

We showed that cell extracts from $N$. gonorrhoeae MS11 were capable of catalysing extensive (>100 bp) reverse transcription on a defined mRNA template. This activity can be explained either by a unique RT present in gonococci or by an intrinsic RT function of DNA polymerase I. The latter explanation is plausible because we showed that purified polymerase I from E. coli was similarly active. This interpretation is consistent with a previous report on the RT function of $E$. coli DNA polymerase I based on a different method (Ricchetti and Buc, 1993, EMBO J 12: 387396). In order to assess whether gonococcal RT activity has a role in gene-conversion processes, we established a genetic system to distinguish between RNA- and DNAmediated recombination. Using this technique, no evidence for the existence of a RNA intermediate was obtained. This observation suggests that mechanisms other than reverse transcription account for the non-reciprocal transformation-independent recombination events between pilin genes in $N$. gonorrhoeae.

We gratefully acknowledge Scott Gray-Owen for helpful comments on the manuscript.

Roland Barten ${ }^{1}$ and Thomas F. Meyer ${ }^{1,2 *}$

${ }^{1}$ Max-Planck-Institut für Biologie, Abteilung

Infektionsbiologie, Spemannstrasse 34, D-72076

Tübingen, Germany.

${ }^{2}$ Max-Planck-Institut für Infektionsbiologie, Abteilung

Molekulare Biologie, Monbijoustrasse 2, D-10117 Berlin, Germany.

* For correspondence (address no. 2).

E-mailsinfbio@mpib-tuebingen.mpg.de; Tel. (7071) 601 221; Fax (7071) 610379.

Received 21 February, 1997; accepted 6 March, 1997.

\section{A quest for symbiosis-specific genes urges itself upon Rhizobium geneticists}

Sir,

Some members of the Rhizobiaceae family are able to oxidize hydrogen $\left(\mathrm{Hup}^{+}\right)$through regulated expression of hydrogen-uptake (hup) genes. This system is particularly useful during symbiosis, when hydrogen is formed by the nitrogenase enzyme complex. Under these conditions, the activity of a hydrogen-oxidizing system is an elegant way of removing hydrogen, which is an inhibitor of the nitrogenase enzyme complex. Interestingly, in $\mathrm{Hup}^{+}$bacteria of the genus Bradyrhizobium, the hup genes are also expressed in free-living cells, when bacteria are shifted to a microaerobic atmosphere containing hydrogen. This might reflect a natural situation, since nitrogenase activity, and thus hydrogen production, in free-living Bradyrhizobium has been reported (Agarwal and Keister, 1983; Appl Environ Microbiol 45: 1592-1601). The transcriptional activator, HoxA, is crucial for expression of hup genes in free-living conditions: hoxA mutants are $\mathrm{Hup}^{-}$in free-living conditions, but are $\mathrm{Hup}^{+}$in symbiotic conditions, where hydrogenase activity amounts to $50 \%$ of the wild-type level (Lambert et al., 1987, Appl Environ Microbiol 53: 422428; Van Soom et al., 1997, Mol Microbiol 23: 967977). This implies that HoxA is functioning in bacteroids but can be replaced by a less efficient and so-far unidentified symbiosis-specific factor. On the other hand, $\mathrm{Hup}^{+}$ bacteria of the genus Rhizobium do not have hydrogenase activity in free-living conditions (Palacios et al., 1990, Mol Gen Genet 221: 363-370). hup gene organization in Rhizobium leguminosarum biovar viciae, is strikingly similar to 
that in Bradyrhizobium, except that the regulatory genes that border the structural and accessory hup genes are missing (hupUV to the 'left', hoxA and hupT to the 'right'). Both the Bradyrhizobium japonicum and $R$. leguminosarum hupSL promoter regions contain a well-conserved integration host factor (IHF)-binding site and an RpoN-dependent consensus promoter sequence (Hidalgo et al., 1992, J Bacteriol 174: 4130-4139; Black and Maier, 1995, Mol Microbiol 16: 405-413). In $R$. leguminosarum, the DNA region $-172 /-87$ relative to the start site of transcription is essential for promoter activity, indicating that this area contains the upstream activating sequence (UAS) of a transcriptional activator (Brito et al., 1995, in Nitrogen Fixation: Fundamentals and Applications. Tikhonovich et al. (eds). Dordrecht: Kluwer Academic Publishers, p. 394). hup genes are expressed in nodules induced by $\mathrm{Hup}^{+} R$. leguminosarum bv. viciae but the nature of the transcriptional activator remains unknown. NifA-dependent expression of the Rhizobium hupSL genes has been demonstrated in Klebsiella pneumoniae hosts, suggesting co-regulation of nif and hup genes in $R$. leguminosarum (Brito et al., 1995, ibid.). As the $R$. leguminosarum bv. viciae hup $S L$ genes are not expressed in microaerobically grown cells, the $R$. leguminosarum NifA alone apparently cannot activate hupSL expression. It appears that, during evolution, the hup structural and accessory genes were highly conserved because of a selective advantage, but the regulatory elements that control the expression of these genes in different bacteria were far less conserved, or even deleted. Activation of hupSL expression by the transcriptional regulator HoxA is absolutely required to obtain hydrogenase activity in free-living $B$. japonicum cells, whereas in bacteroids activation of hupSL expression can be or has been taken over by a symbiosis-specific factor in B. japonicum or $R$. leguminosarum, respectively.

When we compare the nucleotide sequence of the promoter region of the hupSL genes in Bradyrhizobium and Rhizobium using the NMATPUs program (PC-GENE, IntelliGenetics), it is striking that a conserved CT-rich region, with 10 out of 12 identical nucleotides, is present in the upstream regulatory regions that are essential for hupSL promoter activity (Fig. 1). Whether this conserved site is the UAS for the symbiosis-specific factor remains to be demonstrated.

$$
\begin{array}{llll}
\text { Bj UAS } & -131 \text { TTTCCGCTTCTC } & -120 \\
\text { R1 UAS } & -112 \text { TATCCGGTTCTC } & -101
\end{array}
$$

Fig. 1. Nucleotide sequence comparison (NMATPUS; PC-GENE, IntelliGenetics) of part of the upstream regulatory region of the $B$. japonicum (Bj UAS) and R. leguminosarum (RI UAS) hupSL genes. Numbers are relative to the start site of hupSL transcription. The $-149 /-98$ region in $B$. japonicum and the $-172 /-87$ region in $R$. leguminosarum are required for hupSL transcription (for references, see the text).
The differential regulation of hup genes in free-living and symbiotic rhizobia could be explained according to the 'regulatory noise' hypothesis (de Lorenzo and PérezMartin, 1996, Mol Microbio/ 19: 1177-1184), which states that structural and regulatory genes evolve relatively independently and that new transcriptional control systems can evolve because of the non-specificity of pre-existing promoters. It is well known that the differentiation process leading to an intracellularly nitrogen-fixing bacteroid is accompanied by a drastic change in bacterial metabolism and morphology. This implies that different, bacteroid-specific regulatory factors act on bacteroid-specific genes. In the regulatory noise hypothesis, these alternative regulators could interact in an illegitimate way with pre-existing promoter sequences of genes that are normally regulated by other transcriptional factors. When this illegitimate expression has a selective advantage, the newly recruited, sub-optimal transcriptional control unit will be fine-tuned in order to respond predominantly to the new signal.

Another example of differential regulation in free-living versus symbiotic cells can be found in the regulation of $\operatorname{dct} A$, encoding a dicarboxylate permease in Rhizobium meliloti. The transcriptional activator DctD is required for RpoN-dependent expression of the structural genes in free-living cells but can be replaced in bacteroids by a symbiosis-specific regulator, which is neither NifA or NtrC (Ronson et al., 1987, J Bacteriol 169: 2424-2431; Yarosh et al., 1989; Mol Microbiol 3: 813-823; Allaway et al., 1995, FEMS Microbiol Lett 128: 241-245). Differential regulation, albeit more subtle, also exists in the case of nifHDK expression in $R$. meliloti: the same regulator (NifA) binds at different cis-elements in the free-living versus bacteroid state (Better et al., 1985, EMBO J 4: 2419-2424; Ditta et al., 1988, in Molecular Genetics of Plant-Microbe Interactions. Palacios and Verma (eds). St. Paul, Minnesota: APS Press, pp. 109-110; Wang et al., 1991, Mol Microbiol 5: 157-162). The conformation of the UAS and/or of the regulatory protein might be influenced by the symbiotic state, leading to differential affinities of the regulatory protein for certain UASs.

The identification of hitherto unknown factors responsible for symbiosis-specific expression will be of great interest, as it is becoming increasingly apparent that studies on symbiotic regulatory processes ex planta reflect a virtual reality which can be different from real life in the nodule.

Carolien Van Soom and Jos Vanderleyden*

F. A. Janssens Laboratory of Genetics, K.U. Leuven,

W. de Croylaan 42, B-3001 Heverlee, Belgium.

* For correspondence.

E-mail janssens@agr.kuleuven.ac.be; Tel. (16) 322403; Fax (16) 322990.

Received and accepted 27 February, 1997. 